\title{
State Enumeration with Abstract Descriptions of State Machines
}

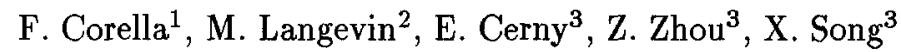 \\ 1 IBM Research, USA, corella@watson.ibm.com \\ ${ }^{2}$ GMD-SET, Germany, langevin@gmd.de \\ 3 Univ. de Montréal, Canada, \{cerny,zhouz,song\}@iro.umontreal.ca
}

\begin{abstract}
We propose a theory of abstract descriptions of state machines in a many-sorted first-order logic with abstract and concrete sorts. State variables containing data values have abstract sorts while control state variables have concrete sorts. Data operations are represented by uninterpreted function symbols. The theory provides a foundation for automated state enumeration methods whose complexity is independent of the width of the datapath, and in particular for methods based on Multiway Decision Grahps (MDGs).
\end{abstract}

\section{Introduction}

Formal verification methods for synchronous hardware can be classified in three categories [16]: 1) verification with a theorem prover, 2) verification using a special calculus, and 3 ) finite state machine (FSM) verification by state enumeration.

In the first kind of methods, design correctness is stated as a theorem to be proved using a theorem proving assistant. Theorem provers use powerful formalisms such as higher-order logic [15] that allow the verification problem to be stated at many levels of abstraction. This makes it possible to verify circuits such as a microprocessor [17] or a hierarchical shared memory subsystem [22] directly at the register-transfer level (RTL). Although the user of the theorem prover has to assist in the proof, some parts can be automated for restricted verification problems, as in $[3,18,23,24]$.

Methods based on special calculi, such as [1,2], were also proposed to automate the verification for a restricted class of circuit behaviors described above the logic level.

State enumeration techniques provide automation for behavioral comparison or model checking, without imposing restrictions on the circuit behaviors, but operate on descriptions at the logic (i.e. binary) level. Recent FSM-based methods exploit the ordered binary decision diagram (OBDD) representation [4] to encode sets of states and to perform an implicit enumeration of the state space, making it possible to verify FSMs with a large number of states [5, 8, 12, 25]. For some specific circuits, these methods achieve linear complexity with respect to the data width. However, these methods are not adequate in general for verifying circuits with datapath because a logic level circuit description is required. These methods do not permit an abstract representation of the circuit, contrary to the approaches based on theorem proving or specific calculi. 
Abstraction techniques that reduce the space of datapath states to a small set of representative states have been proposed as a way of extending the range of verification problems that can be solved by FSM-based automated verification methods [26, 7]. However, the data independence assumption of [26] is highly restrictive, while the method of [7] places a considerable burden on the user of the verification program and does not completely eliminate the dependency on the width of the datapath.

An alternative approach for automatically verifying circuits with datapath is to combine techniques for state enumeration originally devised for FSMs and techniques for symbolic computation with abstract data representations. A data input or data register can then be represented by a single variable of abstract type while data operations are represented by uninterpreted function symbols. In a sense, this bridges the gap between theorem proving and automated verification methods: automated verification becomes possible at a high-level of abstraction, and could be embedded as a specialized decision procedure in a theorem prover.

This alternative approach was first investigated by Langevin and Cerny $[19,20,21]$ and Corella $[9,10]$, who independently developed similar verification methods based on abstract state enumeration (i.e. exploration of the state space using abstract types and uninterpreted function symbols). However, explicit enumeration was used, and this is not adequate for circuits with a complex controller. Recently, we have found a way of combining the strengths of abstract types and decision diagrams for state enumeration: we have conceived a class of decision graphs, called Multiway Decision Graphs (MDGs), that subsume BDDs and accommodate abstract types and uninterpreted function symbols while providing and exploiting structure sharing [11]. Using MDGs we have successfully developed algorithms for reachability analysis, equivalence checking and verification of a microprocessor against its instruction set architecture [28]. These algorithms are based on abstract implicit enumeration of the state space, and are a natural extension of the FSM verification methods that use OBDDs and implicit enumeration.

Abstract types and uninterpreted function symbols have also been used in [6], where a heuristic validity checker is proposed to verify a pipelined implementation against a non-pipelined specification. However this method is of limited scope because it does not allow any state enumeration, implicit or explicit.

In this paper we propose a theory of abstract descriptions of state machines as a foundation for current and future work on verification with abstract types. This theory is not concerned with a new kind of state machine, but rather with a new way of describing state machines, at a higher level of abstraction. While the state machines that we want to verify are ordinary FSM, the abstract descriptions that we propose admit non-finite state machines as models, in addition to their intended, finite interpretations. Note that the motivation for such abstract descriptions is eminently practical: they make it possible to verify a circuit at the RT level without getting bogged down in the details of the gate or transistor level implementation.

In Section 2 we describe the many-sorted first-order logic that we use, which 
contains a built-in convenient distinction between abstract and concrete sorts. In Section 3 we show how abstract descriptions of state machines can be formulated in the logic. Then in Section 4 we show that abstract descriptions permit abstract state enumeration, and in Section 5 we describe MDGs, which provide for implicit abstract enumeration. In the conclusion we outline the scope of the verification methodology and we point out the great opportunities that are open for further research.

\section{Formal Logic}

\subsection{Syntax}

The formal logic that we use is many-sorted first-order logic, with a distinction between abstract sorts and concrete sorts. This distinction, motivated by the distinction between datapath and control in the application domain, is only a matter of convenience; it does not change the expressiveness of the logic.

Concrete sorts have enumerations, while abstract sorts do not. An enumeration is a finite set of constants. For example, there may be a sort BIT with enumeration $\{0,1\}$ or a sort OPCODE with enumeration $\{$ jump_instr,load_instr, ... $\}$. A constant that appears in an enumeration is called an individual constant. Beside individual constants, the vocabulary consists of generic constants, variables, and function symbols (also called operators). Constants and variables have sorts. An $n$-ary function symbol $(n>0)$ has a type $\alpha_{1} \times \ldots \times \alpha_{n} \rightarrow \alpha_{n+1}$, where $\alpha_{1} \ldots \alpha_{n+1}$ are sorts. The (well-typed) terms and their sorts are defined as usual. Generic constants can be viewed as 0-ary function symbols.

The distinction between abstract and concrete sorts leads to a distinction between three kinds of function symbols. Let $f$ be a function symbol of type $\alpha_{1} \times \ldots \times \alpha_{n} \rightarrow \alpha_{n+1}$. If $\alpha_{n+1}$ is an abstract sort then $f$ is an abstract function symbol. Abstract function symbols are used to denote data operations and are uninterpreted. If all the $\alpha_{1} \ldots \alpha_{n+1}$ are concrete, $f$ is a concrete function symbol. Concrete function symbols, and as a special case concrete generic constants, are seldom needed; for the sake of simplicity we shall assume that they are not used. Finally, if $\alpha_{n+1}$ is concrete while at least one of $\alpha_{1} \ldots \alpha_{n}$ is abstract, then we refer to $f$ as a cross-operator. Cross-operators are used to model feedback from the datapath to the control FSM, and are also uninterpreted.

A term $A$ that has no concrete subterms other than individual constants is said to be concretely-reduced; we consider $A$ to be a subterm of itself, hence if $A$ is concretely reduced and of concrete sort it must be an individual constant. A term of the form " $f\left(A_{1}, \ldots, A_{n}\right)$ " where $f$ is a cross-operator and $A_{1} \ldots A_{n}$ are concretely-reduced terms, is a cross-term.

A (well-typed) equation is an expression " $A_{1}=A_{2}$ " where the left-hand side (LHS) $A_{1}$ and the right-hand side (RHS) $A_{2}$ are terms of the same sort $\alpha$. The atomic formulas are the equations, plus $\mathbf{T}$ (truth) and $\mathbf{F}$ (falsity). The formulas of the logic are built from the atomic formulas in the usual way using logical connectives and quantifiers. 


\subsection{Semantics}

An interpretation is a mapping $\psi$ that assigns a denotation to each sort, constant and function symbol and satisfies the following conditions:

1. The denotation $\psi(\alpha)$ of an abstract sort $\alpha$ is a non-empty set.

2. If $\alpha$ is a concrete sort with enumeration $\left\{a_{1}, \ldots, a_{n}\right\}$ then $\psi(\alpha)=\left\{\psi\left(a_{1}\right), \ldots\right.$, $\left.\psi\left(a_{n}\right)\right\}$ and $\psi\left(a_{i}\right) \neq \psi\left(a_{j}\right)$ for $1 \leq i<j \leq n$.

3. If $f$ is a function symbol of type $\alpha_{1} \times \ldots \times \alpha_{n} \rightarrow \alpha_{n+1}$, then $\psi(f)$ is a function from the cartesian product $\psi\left(\alpha_{1}\right) \times \ldots \times \psi\left(\alpha_{n}\right)$ into the set $\psi\left(\alpha_{n+1}\right)$. In particular, if $n=0$ (i.e., $f$ is a generic constant of sort $\left.\alpha_{1}\right), \psi(f) \in \psi\left(\alpha_{1}\right)$.

$X$ being a set of variables, a variable assignment with domain $X$ compatible with an interpretation $\psi$ is a function $\phi$ that maps every variable $x \in X$ of sort $\alpha$ to an element $\phi(x)$ of $\psi(\alpha)$. We write $\Phi_{X}^{\psi}$ for the set of $\psi$-compatible assignments to the variables in $X$. The denotation of a term and the truth or falsity of a formula under an interpretation and a compatible variable assignment are defined as usual. We write $\psi, \phi \models P$ if a formula $P$ denotes truth under an interpretation $\psi$ and a $\psi$-compatible variable assignment $\phi$ to the variables that occur free in $P$, and $\models P$ if $\psi, \phi \models P$ for all such $\psi$ and $\phi$. Two formulas $P$ and $Q$ are logically equivalent iff $\vDash P \Leftrightarrow Q$.

\section{Abstract Descriptions of State Machines}

\subsection{Example}

As a running example we use GCD, a synthesis and verification benchmark. This example is chosen only for illustrative purposes, it is not representative of the level of complexity of state machines that can be verified with abstract types and uninterpreted function symbols.

There are several versions of GCD; we use the traditional version that computes the greatest common divisor of two positive numbers $p_{1}$ and $p_{2}$ by repeated subtraction. The state machine initializes two variables $y_{1}$ and $y_{2}$ with the values $p_{1}$ and $p_{2}$, then repeatedly assigns to the variable with the highest value the difference of the two values, until the two values are the same. When done, the value stored in the two variables is the greatest common divisor. Besides the two data state variables $y_{1}$ and $y_{2}$ there is a control state variable $y_{0}$ which determines two control states $y_{0}=0$ and $y_{0}=1$. When $y_{0}=0$ the machine waits for the two values $p_{1}$ and $p_{2}$ to be presented at two data inputs $x_{1}$ and $x_{2}$, an event which is indicated by a control input $x_{0}$ taking the value 1 . Then $p_{1}$ and $p_{2}$ are loaded into $y_{1}$ and $y_{2}$, and the machine goes to the control state $y_{0}=1$, where it loops until the result has been computed. There is only one output, a data output $z_{0}$ that takes the value 0 while the result is not ready, and produces the GCD when it has been computed.

To obtain an abstract description of this state machine we use a concrete sort BIT with enumeration $\{0,1\}$ and an abstract sort NUM. The abstract sort NUM is uninterpreted: its denotation is any non-empty set, finite or infinite. Its 
intended denotation is of course the set of $n$-bit numbers, where $n$ is the width of the datapath. But the fact that NUM is abstract means that we do not need to specify $n$, and hence that the time and space required to verify the circuit will be independent of the width of the datapath.

The input variable $x_{0}$ and the state variable $y_{0}$, which are control variables, are of concrete sort BIT. On the other hand, the input variables $x_{1}$ and $x_{2}$, the state variables $y_{1}$ and $y_{2}$, and the output variable $z_{0}$ are of abstract sort NUM. We also use three next-state variables, $y_{0}^{\prime}$ of sort BIT, and $y_{1}^{\prime}$ and $y_{2}^{\prime}$ of sort NUM.

To denote subtraction, we use an abstract function symbol sub of type NUM $\times$ $\mathrm{NUM} \rightarrow$ NUM. The function symbol $s u b$ is uninterpreted, i.e. it may denote, under an interpretation $\psi$, any function from $\psi(\mathrm{NUM})^{2}$ into $\psi(\mathrm{NUM})$. On the negative side, this means that the verification procedure does not know that $s u b$ represents subtraction; on the positive side, it means that we do not have to describe the details of the subtraction operation.

The control FSM needs two pieces of information from the datapath: whether $y_{1}=y_{2}$, to terminate the loop, and whether $y_{1}<y_{2}$, to decide which subtraction to make and which value to replace. This feedback from the datapath is modeled using two function symbols $e q$ and $l t$ of type NUM $\times$ NUM $\rightarrow$ BIT. Since BIT is concrete while NUM is abstract, $e q$ and $l t$ are cross-operators. The intended denotation of $e q$ (resp. $l t$ ) is the function that maps an ordered pair of $n$-bit numbers to 1 if they are equal (resp. if the first is less than the second), or to 0 otherwise. However, cross-operators, like abstract function symbols, are uninterpreted; $e q$ and $l t$ can denote arbitrary functions from $\psi(\mathrm{NUM})^{2}$ into $\psi(\mathrm{BIT})$.

To obtain a formula that describes the transition relation of the state machine we distinguish cases as needed according to the values of the variables $x_{0}$ and $y_{0}$ as well as the two feedback values. Informally speaking, in the waiting state $y_{0}=0$, if $x_{0}=1$ then the numbers whose GCD is to be computed are read in, and the machine goes to the looping state $y_{0}^{\prime}=1, y_{1}^{\prime}=x_{1}, y_{2}^{\prime}=x_{2}$. On the other hand if $x_{0}=0$ then nothing changes. In the looping state $y_{0}=1$ it is not necessary to distinguish cases according to the value of $x_{0}$, but we must distinguish cases according to the feedback values. If $e q\left(y_{1}, y_{2}\right)=1$ then the computation is finished and the machine goes back to state $y_{0}^{\prime}=0$, with no other changes: $y_{0}^{\prime}=0, y_{1}^{\prime}=y_{1}, y_{2}^{\prime}=y_{2}$. If $e q\left(y_{1}, y_{2}\right)=0$ then we must consider the value of $l t\left(y_{1}, y_{2}\right)$. If it is 0 , then $y_{0}^{\prime}=1, y_{1}^{\prime}=y_{1}-y_{2}, y_{2}^{\prime}=y_{2}$ while if it is 1 then $y_{0}^{\prime}=1, y_{1}^{\prime}=y_{1}, y_{2}^{\prime}=y_{2}-y_{1}$. Thus the transition relation can be described by the following formula:

$$
\begin{aligned}
\left(\left(y_{0}=0\right)\right. & \left.\wedge\left(x_{0}=0\right) \wedge\left(y_{0}^{\prime}=0\right) \wedge\left(y_{1}^{\prime}=y_{1}\right) \wedge\left(y_{2}^{\prime}=y_{2}\right)\right) \vee \\
\left(\left(y_{0}=0\right)\right. & \left.\wedge\left(x_{0}=1\right) \wedge\left(y_{0}^{\prime}=1\right) \wedge\left(y_{1}^{\prime}=x_{1}\right) \wedge\left(y_{2}^{\prime}=x_{2}\right)\right) \vee \\
\left(\left(y_{0}=1\right)\right. & \wedge\left(e q\left(y_{1}, y_{2}\right)=0\right) \wedge\left(l t\left(y_{1}, y_{2}\right)=0\right) \wedge \\
\left(y_{0}^{\prime}=1\right) & \left.\wedge\left(y_{1}^{\prime}=\operatorname{sub}\left(y_{1}, y_{2}\right)\right) \wedge\left(y_{2}^{\prime}=y_{2}\right)\right) \vee \\
\left(\left(y_{0}=1\right)\right. & \wedge\left(e q\left(y_{1}, y_{2}\right)=0\right) \wedge\left(l t\left(y_{1}, y_{2}\right)=1\right) \wedge \\
\left(y_{0}^{\prime}=1\right) & \left.\wedge\left(y_{1}^{\prime}=y_{1}\right) \wedge\left(y_{2}^{\prime}=\operatorname{sub}\left(y_{2}, y_{1}\right)\right)\right) \vee \\
\left(\left(y_{0}=1\right)\right. & \left.\wedge\left(e q\left(y_{1}, y_{2}\right)=1\right) \wedge\left(y_{0}^{\prime}=0\right) \wedge\left(y_{1}^{\prime}=y_{1}\right) \wedge\left(y_{2}^{\prime}=y_{2}\right)\right)
\end{aligned}
$$

This formula can be derived from a structural description of a circuit that implements the state machine, the circuit being a network of components such as 
registers, gates, comparators, and arithmetic units, or from a behavioral specification written in an algorithmic hardware description language.

The output relation is the relationship between the value of the output $z_{0}$ and the values of the inputs and state variables. It can be described by the following formula, where zero is a generic constant of abstract sort NUM:

$$
\begin{aligned}
& \left(\left(y_{0}=0\right) \wedge\left(z_{0}=y_{1}\right)\right) \vee \\
& \left(\left(y_{0}=1\right) \wedge\left(e q\left(y_{1}, y_{2}\right)=0\right) \wedge\left(z_{0}=z e r o\right)\right) \vee \\
& \left(\left(y_{0}=1\right) \wedge\left(e q\left(y_{1}, y_{2}\right)=1\right) \wedge\left(z_{0}=y_{1}\right)\right)
\end{aligned}
$$

It remains to describe the set of initial states. There could be a single initial state, where all three state variables take the value 0 . This can be described by the formula $\left(y_{0}=0\right) \wedge\left(y_{1}=z e r o\right) \wedge\left(y_{2}=z e r o\right)$. Or any waiting state could be an initial state. The set of initial states would then be described by the formula

$$
\left(y_{0}=0\right) \wedge\left(y_{1}=w_{1}\right) \wedge\left(y_{2}=w_{2}\right)
$$

where $w_{1}$ and $w_{2}$ are auxiliary variables of sort NUM.

\subsection{General Case}

Given two disjoint sets of variables $U$ and $V$, a directed formula (DF) of type $U \rightarrow V$ is a formula $P$ in disjunctive normal form (DNF) where each disjunct is a conjunction of equations, and each equation is of one of the following forms:

1. $A=a$, where $A$ is cross-term of (concrete) sort $\alpha$ containing no variables other than elements of $U$, and $a$ is an individual constant in the enumeration of $\alpha$;

2. $t=a$, where $t \in U \cup V$ is a variable of concrete sort $\alpha$ and $a$ is an individual constant in the enumeration of $\alpha$; or

3. $v=A$, where $v \in V$ is a variable of abstract sort $\alpha$ and $A$ is a concretely reduced term of type $\alpha$ containing no variables other than elements of $U$,

with each LHS appearing in at most one equation, and every abstract variable $v \in V$ appearing as the LHS of one equation in each of the disjuncts.

The requirements that $A$ be a cross-term in case 1 , and $A$ be concretely reduced in case 3 mean that every concrete term appearing in one of the equations other than as the LHS of the equation must be an individual constant. This can always be achieved by case splitting. In particular, every variable that occurs in the LHS of case 1 or the RHS of case 3 must be abstract. We refer to those variables as the secondary variables of $P$. A primary variable of $P$, on the other hand, is one that appears as the LHS of case 2 or 3 . Since a secondary variable is an abstract $U$ variable while a primary variable is a concrete $U$ variable or a $V$ variable, no variable can be both.

We use DFs for two distinct purposes: to represent relations (viz. the transition and output relations) and to represent sets (viz. sets of sets as well as sets of input vectors and output vectors). To see how a DF can represent a set, note 
that a variable assignment $\phi$ with domain $V$ compatible with a given interpretation $\psi$ can be viewed as a vector of values, indexed by the variables in $V$. Let $P$ be a DF of type $U \rightarrow V$, where $U$ only contains abstract variables. Then $P$ represents the set of vectors

$$
\operatorname{Set}^{\psi}(P)=\left\{\phi \in \Phi_{V}^{\psi} \mid \psi, \phi \vDash(\exists U) P\right\} .
$$

If $\phi \in \operatorname{Set}^{\psi}(P)$ we say that $P$ covers $\phi$.

From now on we shall use a denumerable set $W$ of auxiliary abstract variables from which fresh abstract variables can be obtained as needed. An abstract description of a state machine is a tuple $D=\left(X, Y, Z, F_{I}, F_{T}, F_{O}\right)$ where:

1. $X, Y$ and $Z$ are finite sets of variables (the input, state, and output variables respectively), which are pairwise disjoint as well as disjoint from $W$. Let $Y^{\prime}$ be the set of next-state variables, disjoint from $X \cup Y \cup Z \cup W$, each next-state variable being obtained, for example, by priming the corresponding presentstate variables; and let $\eta$ be the function that maps each state variable to the corresponding next-state variable.

2. $F_{I}$ is a DF of type $W \rightarrow Y$ (description of the set of initial states).

3. $F_{T}$ is a DF of type $(X \cup Y) \rightarrow Y^{\prime}$ (description of the transition relation).

4. $F_{O}$ is a DF of type $(X \cup Y) \rightarrow Z$ (description of the output relation).

For each interpretation $\psi$ there is exactly one state machine that satisfies such a description, viz. the tuple $M=\left(\Phi_{X}^{\psi}, \Phi_{Y}^{\psi}, \Phi_{Z}^{\psi}, S_{I}, R_{T}, R_{O}\right)$ where:

1. $\Phi_{X}^{\psi}$ is the set of all $\psi$-compatible assignments to the variables in $X$, i.e. the set of all input vectors, the input alphabet of the state machine; similarly, $\Phi_{Y}^{\psi}$ is the set of states and $\Phi_{Z}^{\psi}$ the output alphabet of the machine.

2. $S_{I}=\operatorname{Set}^{\psi}\left(F_{I}\right)$ is the set of initial states.

3. $R_{T}=\left\{\left(\phi, \phi^{\prime}, \phi^{\prime \prime}\right) \in \Phi_{X}^{\psi} \times \Phi_{Y}^{\psi} \times \Phi_{Y}^{\psi} \mid \psi, \phi \cup \phi^{\prime} \cup \phi^{\prime \prime} \circ \eta \models F_{T}\right\}$ is the transition relation.

4. $R_{O}=\left\{\left(\phi, \phi^{\prime}, \phi^{\prime \prime}\right) \in \Phi_{X}^{\psi} \times \Phi_{Y}^{\psi} \times \Phi_{Z}^{\psi} \mid \psi, \phi \cup \phi^{\prime} \cup \phi^{\prime \prime} \vDash F_{O}\right\}$ is the output relation.

\section{Abstract State Enumeration}

\subsection{Image Computation}

Let $D=\left(X, Y, Z, F_{I}, F_{T}, F_{O}\right), \eta, \psi$, and $M=\left(\Phi_{X}^{\psi}, \Phi_{Y}^{\psi}, \Phi_{Z}^{\psi}, S_{I}, R_{T}, R_{O}\right)$ be as above. Let $W_{1}$ and $W_{2}$ be disjoint subsets of $W$. Let $Q$ be a DF of type $W_{1} \rightarrow Y$ representing a set of states $S_{Q}=S e t^{\psi}(Q)$, and $P$ a DF of type $W_{2} \rightarrow X$ representing a set of input vectors $S_{P}=S e t^{\psi}(P)$. The image $S_{Q^{\prime}}$ is the set of states reachable in one step from states in $S_{Q}$ and inputs in $S_{P}$. That is, $\phi^{\prime \prime} \in S_{Q^{\prime}}$ iff

$$
\psi, \phi \cup \phi^{\prime} \cup \phi^{\prime \prime} \circ \eta \models\left(\exists W_{2}\right) P \wedge\left(\exists W_{1}\right) Q \wedge F_{T}
$$

for some $\phi \in \Phi_{X}^{\psi}$ and some $\phi^{\prime} \in \Phi_{Y}^{\psi}$ or, in other words, $\phi^{\prime \prime} \in S_{Q^{\prime}}$ iff

$$
\psi, \phi^{\prime \prime} \circ \eta \vDash(\exists X)(\exists Y)\left(\left(\exists W_{2}\right) P \wedge\left(\exists W_{1}\right) Q \wedge F_{T}\right) .
$$


But the $W_{1}$ variables only occur in $Q$ and the $W_{2}$ variables only occur in $P$. Therefore $\phi^{\prime \prime} \in S_{Q^{\prime}}$ iff

$$
\psi, \phi^{\prime \prime} \circ \eta \vDash(\exists W)(\exists X)(\exists Y)\left(P \wedge Q \wedge F_{T}\right) .
$$

We can obtain a DF $Q^{\prime}$ of type $W \rightarrow Y$, such that $S_{Q^{\prime}}=\operatorname{Set} t^{\psi}\left(Q^{\prime}\right)$, by transforming the formula

$$
(\exists X)(\exists Y)\left(P \wedge Q \wedge F_{T}\right)
$$

into a logically equivalent DF of type $W \rightarrow Y^{\prime}$, and then renaming $Y^{\prime}$ into $Y$.

Put (3) in DNF by distributing $\wedge$ over $\vee$. Each disjunct in the DNF is a conjunction of equations of the following kinds:

1. $y=a$ (resp. $x=a$ ) where $y \in Y$ (resp. $x \in X$ ) is concrete and $a$ is an individual constant. Such equations may come from $Q$ (resp. $P$ ) or from $F_{T}$. If there are two with the same variable but different constants, discard the disjunct, since it is contradictory according to the semantics of Section 2.2 .

2. $y=A$ (resp. $x=A$ ) where $y \in Y$ (resp. $x \in X$ ) is abstract and $A$ is a concretely reduced term containing no variables other than auxiliary variables. Such equations come from $Q$ (resp. $P$ ), and there is one for each abstract variable $y \in Y$ (resp. $x \in X$ ). The LHS of the equation is the primary occurrence of the variable in the disjuncts, other occurrences being secondary; the RHS $A$ is the symbolic value of the variable in the disjunct.

3. $A=a$, where $A$ is a cross-term containing no variables other than the auxiliary variables $W_{1}$ (resp. $W_{2}$ ). Such equations come from $Q$ (resp. $P$ ).

4. $y^{\prime}=a$ where $y^{\prime} \in Y^{\prime}$ is concrete and $a$ is an individual constant. Such an equation comes from $F_{T}$.

5. $y^{\prime}=A$ where $y^{\prime} \in Y^{\prime}$ is abstract and $A$ is a concretely reduced term containing no variables other than abstract $X$ or $Y$ variables. Such an equation comes from $F_{T}$. By context sensitive substitution, replace each $x \in X$ or $y \in Y$ that occurs in $A$ with its symbolic value in the disjunct. This results in an equation of the form $y^{\prime}=B$, where $B$ is a concretely reduced term containing no variables other than auxiliary variables.

6. $A=a$ where $A$ is a cross-term containing no variables other than abstract $X$ or $Y$ variables and $a$ is an individual constant. Such an equation comes from $F_{T}$. By context sensitive substitution, replace each $x \in X$ or $y \in Y$ that occurs in $A$ with its symbolic value in the disjunct. This results in an equation of the form $B=a$, where $B$ is a concretely reduced term containing no variables other than auxiliary variables. Then, discard the disjunct if there is two equations with the same cross-term but different constants.

After eliminating duplicated equations, each disjunct ${ }^{4}$ in the DNF has at most one occurrence of each $v \in X \cup Y$, and that occurrence is the LHS of an equation: discard the equation, since $v$ is existentially quantified. Then discard the quantifiers $(\exists X)(\exists Y)$, since there remain no occurrences of input or state variables in the formula. The DF $Q^{\prime}$ is finally obtained by replacing each next-state variable with the corresponding present-state variable.

\footnotetext{
${ }^{4}$ There could be no disjuncts left, in which case the resulting formula is F.
} 


\subsection{Example}

As an example of these transformations, consider again the GCD state machine of Section 3.1. In this case $X=\left\{x_{0}, x_{1}, x_{2}\right\}, Y=\left\{y_{0}, y_{1}, y_{2}\right\}, Y^{\prime}=\left\{y_{0}^{\prime}, y_{1}^{\prime}, y_{2}^{\prime}\right\}$, and $Z=\left\{z_{0}\right\}$. The transition relation is represented by the DF (1) of type $(X \cup Y) \rightarrow Y^{\prime}$. Let the set of initial states be the one represented by (2).

Suppose that we want to compute the set of states reachable in one step from the set of initial states, with no constraints on the input vectors. To represent the set of input vectors we use the formula

$$
\left(x_{1}=w_{3}\right) \wedge\left(x_{2}=w_{4}\right)
$$

where $w_{3}$ and $w_{4}$ are auxiliary variables of abstract sort NUM.

To compute the image we start with the formula (3) where $P$ is (4), $Q$ is (2), and $F_{T}$ is (1). The DNF of this formula is composed of five disjuncts. Three of these disjuncts contain both equations $y_{0}=0$ and $y_{0}=1$ and can be discarded. After discarding duplicate equations we get the formula:

$$
\begin{aligned}
& \left(\exists x_{0}\right)\left(\exists x_{1}\right)\left(\exists x_{2}\right)\left(\exists y_{0}\right)\left(\exists y_{1}\right)\left(\exists y_{2}\right) \\
& \left(\left(\left(x_{1}=w_{3}\right) \wedge\left(x_{2}=w_{4}\right) \wedge\left(y_{0}=0\right) \wedge\left(y_{1}=w_{1}\right) \wedge\left(y_{2}=w_{2}\right) \wedge\right.\right. \\
& \left.\left(x_{0}=0\right) \wedge\left(y_{0}^{\prime}=0\right) \wedge\left(y_{1}^{\prime}=y_{1}\right) \wedge\left(y_{2}^{\prime}=y_{2}\right)\right) \vee \\
& \left(\left(x_{1}=w_{3}\right) \wedge\left(x_{2}=w_{4}\right) \wedge\left(y_{0}=0\right) \wedge\left(y_{1}=w_{1}\right) \wedge\left(y_{2}=w_{2}\right) \wedge\right. \\
& \left.\left.\left(x_{0}=1\right) \wedge\left(y_{0}^{\prime}=1\right) \wedge\left(y_{1}^{\prime}=x_{1}\right) \wedge\left(y_{2}^{\prime}=x_{2}\right)\right)\right)
\end{aligned}
$$

Then we replace every secondary occurrence of an abstract state or input variable with the symbolic value of the variable in the disjunct, after which we discard the equations containing the primary occurrences, obtaining the formula:

$$
\begin{aligned}
& \left(\exists x_{0}\right)\left(\exists x_{1}\right)\left(\exists x_{2}\right)\left(\exists y_{0}\right)\left(\exists y_{1}\right)\left(\exists y_{2}\right) \\
& \left(\left(\left(y_{0}^{\prime}=0\right) \wedge\left(y_{1}^{\prime}=w_{1}\right) \wedge\left(y_{2}^{\prime}=w_{2}\right)\right) \vee\left(\left(y_{0}^{\prime}=1\right) \wedge\left(y_{1}^{\prime}=w_{3}\right) \wedge\left(y_{2}^{\prime}=w_{4}\right)\right)\right)
\end{aligned}
$$

Finally we remove quantifiers of state and input variables and replace next-state variables with present-state variables:

$$
\left(\left(y_{0}=0\right) \wedge\left(y_{1}=w_{1}\right) \wedge\left(y_{2}=w_{2}\right)\right) \vee\left(\left(y_{0}=1\right) \wedge\left(y_{1}=w_{3}\right) \wedge\left(y_{2}=w_{4}\right)\right)
$$

In this particular case, the resulting DF represents all the possible states.

\subsection{Subsumption}

Given a set of state variables $Y$, a state description is defined as a DF of type $W \rightarrow Y$ having a single disjunct. Every DF of type $W \rightarrow Y$ is a disjunction of state descriptions, and the set of states covered by the DF is the union of the sets of states covered by the state descriptions.

A state description is complete iff every concrete state variable $y$ (as every abstract state variable) appears as the LHS of one equation. Note that a state description, even if complete, can cover more than one state, and indeed it can cover an arbitrarily large set of states, since the term $A$ in an equation $y=$ $A$ where $y$ is an abstract state variable may contain auxiliary variables. In a 
complete state description, the equations of the form $y=a$ and $y=A$ can be viewed as comprising an assignment of symbolic values to the state variables, while the equations $A=a$ can be viewed as imposing constraints on the auxiliary variables that occur in the symbolic values.

We say that a state description $D_{1}$ is subsumed by a state description $D_{2}$ iff there exists a substitution $\theta$ with $\operatorname{dom}(\theta) \subseteq W$ such that every equation in the state description $D_{2} \cdot \theta$ obtained by applying $\theta$ to $D_{2}$ is also an equation in the state description $D_{1}$.

Let $P$ be a DF of type $W \rightarrow Y$ and $D$ a state description that is a disjunct of $P$. If $A$ (resp. $y$ ) is a cross-term (resp. state variable) of concrete sort $\alpha$ with enumeration $\left\{a_{i}\right\}_{1 \leq i \leq n}$, and there is no equation of the form $A=a_{i}$ (resp. $\left.y=a_{i}\right)$ in $D$, a $\mathrm{DF}^{\prime} P^{\prime}$ logically equivalent to $P$ and also of type $W \rightarrow Y$ can be obtained by replacing the disjunct $D$ with the collection of disjuncts $D \wedge\left(A=a_{i}\right)$ (resp. $y=a_{i}$ ), $1 \leq i \leq n$. We say that $P^{\prime}$ is derived from $P$ by case splitting. More generally, we say that $P^{\prime}$ is derived from $P$ by case splitting if it can be obtained by one or more of such case splitting operations.

Given two DFs $P$ and $Q$ of type $W \rightarrow Y, P$ is said to be subsumed by $Q$ iff there exists a third DF $P^{\prime}$ of type $W \rightarrow Y$, derivable from $P$ by case splitting, such that every state description in $P^{\prime}$ is subsumed by some state description in $Q$. If $P$ is subsumed by $Q$, then $\vDash(\exists W) P \Rightarrow(\exists W) Q$ and thus, for any interpretation $\psi, \operatorname{Set}^{\psi}(P) \subseteq \operatorname{Set}^{\psi}(Q)$.

Let $P, P^{\prime}, Q$ be DFs of type $W \rightarrow Y . P^{\prime}$ is said to be derivable from $P$ by pruning-by-subsumption w.r.t. $Q$ iff the state descriptions in $P^{\prime}$ are a subset of those in $P$, and the state descriptions in $P$ which are not in $P^{\prime}$ are subsumed by $Q$. When this is the case, $\vDash(\exists W) P \vee(\exists W) Q \Leftrightarrow(\exists W) P^{\prime} \vee(\exists W) Q$ and $\vDash P^{\prime} \Rightarrow P$. If $P^{\prime}=\mathrm{F}$ is derivable from $P$ by pruning-by-subsumption w.r.t. $Q$, then $P$ is subsumed by $Q$ and $\operatorname{Set}^{\psi}(P) \subseteq \operatorname{Set}^{\psi}(Q)$. We describe a pruning-bysubsumption algorithm in [11].

\subsection{Reachability Analysis and Related Verification Methods}

We have seen how to compute the image of a set by the transition relation, and how to check whether a set of states is a subset of another set of states. Computing the union of two sets of states is simply a matter of taking the disjunctions of their representations. With these ingredients it is possible to implement a number of verification methods. The set of reachable states can be computed iteratively from the set of initial states using image computations and disjunction, and using a subsumption check to terminate the iteration. It is possible to check whether a certain condition on the outputs holds for all the reachable states. It is possible to check the equivalence of two state machines by checking that they produce the same outputs in all the reachable states of the product machine. It is possible to verify a conventional (non-pipelined) microprocessor against its instruction set architecture, by forming the product of a state machine derived from the implementation and a state machine derived from the architecture and duly synchronized with the implementation. It is possible to 
verify an implementation against an algorithmic specification. For details on all this see $[9,10,19,20,21,27,28]$.

However there are cases where the set of reachable states is not representable by a finite DF of type $W \rightarrow Y$, and in such cases the above verification procedures will not terminate. For example, consider the case of a microprocessor having a program counter whose initial value is 0 , denoted by a generic constant zero of abstract sort. An instruction that does not change the flow of control increments the program counter; assume that an abstract function symbol inc is used to represent this. A DF $P_{k}$ of type $W \rightarrow Y$ representing the set of states reachable in up to $k$ steps must have at least $k$ disjuncts (state descriptions), containing the equations $y_{\mathrm{pc}}=$ zero, $y_{\mathrm{pc}}=\operatorname{inc}(z e r o), y_{\mathrm{pc}}=i n c($ inc $(z e r o)), \ldots, y_{\mathrm{pc}}=i n c^{k}(z e r o)$. A DF representing all the reachable states would require an infinite number of disjuncts.

In some cases non-termination can be avoided by generalizing the set of initial states so as to obtain a larger set of reachable states that is representable by a finite DF, while still satisfying the condition to be verified. An important case in which this method is applicable is that of conventional microprocessors and similar circuits that exhibit a cyclic behavior. When comparing two state machines derived from two implementations of a processor, or from an implementation and a specification, the initial state of the product machine can be arbitrary, subject only to two constraints: (i) each machine's control state is the one where the instruction cycle begins, and (ii) the corresponding visible registers in both machines have the same initial values. Then the set of reachable states usually has a finite representation because, informally speaking, after an instruction has been executed the product machine goes to a state that is a special case of this initial state. (In the case discussed above, non-termination would be avoided by letting the value of the program counter be represented by a variable rather than a constant, which would allow the subsumption check to succeed.)

We do not know at present whether the problem of verifying that a certain condition holds is decidable when using abstract sorts, completely uninterpreted function symbols and abstract descriptions of state machines.

\section{Abstract Implicit Enumeration with MDGs}

In the previous section we have described verification methods in terms of manipulations of directed formulas, without concern for efficiency. For the automated verification of complex designs the computations must be done efficiently, using appropriate data structures to represent the various kinds of formulas.

In the early work on abstract state enumeration [9, 10, 19, 20, 21], a set of states was represented by a collection of state descriptions, while the transition relation was specified using "if-then-else" or "case" expressions. In [19, 20, 21], the state descriptions are not necessarily complete, and the transition relation is structured as an EOBDD [21] with structure sharing; however no structure sharing is used for representing sets of states. 
We refer to techniques where state descriptions are enumerated one at a time as abstract explicit enumeration because they resemble traditional FSM explicit enumeration techniques, with the essential difference, however, that a state description can cover a large set of states rather than a single state. We have developed a technique called abstract implicit enumeration where decision graphs are used to represent sets of states as well as the transition and output relations. This technique uses Multiway Decision Graphs (MDGs), a class of decision graphs that subsumes the class of OBDDs while accommodating abstract types and uninterpreted function symbols [11]. Structure sharing in MDGs makes it possible to combine many abstract state descriptions into a single compact graph in the same way as OBDDs can combine many binary state descriptions in a single graph, achieving exponential compaction factors.

Given two disjoint sets of variables $U$ and $V$, an MDG of type $U \rightarrow V$ is a directed acyclic graph (DAG) with one root where

- Every leaf node is labeled by the formula $\mathbf{T}$, except if the graph has a single node, which may be labeled $\mathbf{T}$ or $\mathbf{F}$, and every internal node $N$ falls in one of the following categories:

1. $N$ is labeled by a variable of concrete sort $\alpha$ that is an element of $U \cup V$, and the edges that issue from $N$ are labeled by individual constants in the enumeration of $\alpha$ (not necessarily all of them); or

2. $N$ is labeled by a cross-term of (concrete) sort $\alpha$ containing no variables other than elements of $U$, and the edges that issue from $N$ are labeled by individual constants in the enumeration of $\alpha$ (not necessarily all of them); or

3. $N$ is labeled by a variable of abstract sort $\alpha$ that is an element of $V$ and the edges that issue from $N$ are labeled by concretely reduced terms of type $\alpha$ containing no variables other than elements of $U$,

- Along every path, every abstract variable in $V$ appears exactly once as a node label and there are no duplicate node labels.

Additional well-formedness conditions, analogous to the ordering and minimality conditions of OBDDs, can be found in [11].

An MDG $G$ represents a formula defined inductively as follows: (i) if $G$ consists of a single leaf node labeled by a formula $P$ (which can only by $\mathbf{T}$ or F), then $G$ represents $P$; (ii) if $G$ has a root node labeled $A$ with edges labeled $B_{1} \ldots B_{n}$ leading to subgraphs $G_{1}^{\prime} \ldots G_{n}^{\prime}$, and if each $G_{i}^{\prime}$ represents a formula $P_{i}$, then $G$ represents the formula $\bigvee_{1<i<n}\left(\left(A=B_{i}\right) \wedge P_{i}\right)$. An MDG of type $U \rightarrow V$ is a representation of a DF of type $U \rightarrow V$, and the representation is canonical: it is shown in [11] that, if two MDGs represent logically equivalent formulas, then they are isomorphic.

In the Boolean domain MDGs reduce to OBDDs, in the sense that there is a bijective correspondence between MDGs whose internal nodes are all labeled by Boolean variables and OBDDs; the MDG is obtained from the OBDD by removing the edges leading to the leaf node $\mathbf{F}$, and the OBDD is obtained from the MDG by restoring those edges, which can be done in only one way. 
We have implemented in Prolog an MDG package including algorithms for disjunction, relational product (image computation), and pruning by subsumption. We have used these algorithms to implement reachability analysis, equivalence checking, and verification of a conventional microprocessor against its instruction set architecture. We have experimented with two processors previously used as verification benchmarks, the Tamarack-3 and CompAs; detailed experimental results can be found in [27,28]. To our knowledge, the only successful verification of one of these processors by OBDD methods (the Tamarack) took 370 seconds [13], while we have been able to verify it in just 6 seconds.

\section{Conclusion}

We have proposed a notion of abstract descriptions of state machines as a theoretical foundation for a verification methodology that makes it possible to verify sequential circuits automatically at the RT level, using abstract types and uninterpreted function symbols. We have shown how two state enumeration techniques, the early technique of abstract explicit enumeration based on state descriptions and the newer technique of abstract implicit enumeration using MDGs, can be explained in terms of the proposed theory.

One benefit of the theory is that it helps clarify the scope of the verification methodology. Since the abstract function symbols and the cross-operators are both uninterpreted, the correctness statement must be true for any interpretation of such symbols. The implementation must be an RTL description of the circuit where components that perform data operations or provide feedback from the datapath are black boxes named by uninterpreted function symbols. The specification can be another circuit known to be correct, or an instruction set architecture, or a property of the circuit to be verified; but in any case it must be couched in terms of the same set of uninterpreted function symbols.

Consider again the GCD example. Suppose that we wish to establish that a gate-level implementation indeed produces the GCD of two 32-bit numbers. To use the abstract verification methodology the problem could be decomposed into the following subtasks: (i) verify that the algorithm for computing the GCD of two numbers by repeated subtraction is correct; (ii) verify that the algorithm is correctly implemented by an RTL description of the circuit including a subtraction unit, a comparator for equality $(=)$, and a comparator for inequality $(<)$, viewed as black boxes; and (iii) verify that the gate-level implementations of the subtraction unit and the comparators are correct. Task (ii) can be accomplished by the abstract verification methodology, ${ }^{5}$ while tasks (i) and (iii) are outside of its scope. Another task that would be outside of the scope of the methodology

${ }^{5}$ We have not yet implemented a procedure for verification against an algorithmic specification with MDGs, but GCD has been verified in 1 second for any width of the datapath by the explicit enumeration method of [9]. By contrast, in the experiments reported in [14], verification of a 4-bit version of GCD took 2200 seconds and verification of an 8 -bit version was impossible. 
would be to compare two RTL implementations of GCD that use different data operations, e.g. one that uses subtraction, and one that uses modulus.

While the scope may seem narrow, automated abstract verification is a key element of an overall verification strategy, because it makes it possible to automate tasks that might otherwise have to be performed using a theorem prover.

The fact that abstract function symbols and cross-operator are uninterpreted in the abstract verification methodology does not rule out using partial information about them. For example, while not using a definition of the cross-operator $l t$, it may desirable to use in the verification process the fact that less-than is a transitive relation. And while not making use of a definition of multiplication, it may help to know that 1 is its unit element. For example, using this latter fact might be required to verify an optimization that bypasses a multiplier when one of the operands is 1 . To effectively use this fact we can formulate it as the algebraic equations $1 \times x=x$ and $x \times 1=x$ which would provide partial information about the abstract operator $x$ and could be used as a rewrite rules.

We have actually implemented a conditional rewriting algorithm for MDGs and we have been using it successfully in some experiments that we shall report later. But so far we have only scratched the surface of a very rich field of research, which intersects the field of program verification and abstract data types.

\section{References}

1. Bartsch, A., Eveking, H., Faerber, H.-J., Keletatchew, M., Pinder, J., Schellin, U.: LOVERT - A logic verifier of register-transfer level description. In L. Claesen, ed., Proc. of Int. Work. on Applied Formal Methods for Correct VLSI Design, North-Holland, 1989.

2. Borrione, D., Camurati, P., Prinetto, P., Paillet, J.-L.: Functional approaches applied to microprogrammed architectures. Int. Journal of Computer Aided VLSI Design, 2(4):339-358, 1990.

3. Bronstein, A.: MLP: String-Functional Semantics and Boyer-Moore Mechanization for the Formal Verification of Synchronous Circuits. PhD thesis, Stanford University, Department of Computer Science, 1989.

4. Bryant, R. E.: Graph-Based Algorithms for Boolean Function Manipulation. IEEE Trans. on Comp., 35(8):677-691, 1986.

5. Burch, J. R., Clarke, E. M., Long, D. E., McMillan, K. L., Dill, D. L.: Symbolic model checking for sequential circuit verification. IEEE Trans. on CAD, 13(4):401424, 1994.

6. Burch, J.R., Dill, D.L.: Automatic verification of pipelined microprocessor control. In Proc. of Work. on Computer-Aided Verification, 1994.

7. Clarke, E.M., Grumberg, O., Long, D.E.: Model checking and abstraction. In Proc. of Symp. on Principles of Programming Languages, 1992.

8. Cho, H., Hachtel, G.D., Jeong, S.-W., Plessier, B., Schwarz, E., Somenzi, F.: ATPG Aspects of FSM Verification. In Proc. of ICCAD, pp. 134-137, 1990.

9. Corella, F.: Automated high-level verification against clocked algorithmic specifications. In D. Agnew, et al., ed., Proc. of CHDL, North-Holland, 1993.

10. Corella, F.: Automated verification of behavioral equivalence for microprocessors. IEEE Trans. on Comp., 43(1):115-117, 1994. 
11. Corella, F., Zhou, Z., Song, X., Langevin, M., Cerny, E.: Multiway decision graphs for automated hardware verification. Technical Report RC19676, IBM T. J. Watson Research Center, July 1994.

12. Coudert, O., Madre, J. C.: A Unified Framework for the Formal Verification of Sequential Circuits. In Proc. of ICCAD, 1990.

13. Cyrluk, D., Rajan, S., Shankar, N., Srivar, M.K.: Effective theorem proving for hardware verification. In Int. Conf. on Theorem Provers in Circuit Design, 1994.

14. Filkorn, T., Payer, M., Warkentin, P.: Symbolic verification of high-level synthesis results from CALLAS. In Proc. of High-Level Synthesis Workshop, 1993.

15. Gordon, M.J.C., Melham, T.F.: An Introduction to HOL. Cambridge University Press, 1993.

16. Gupta, A.: Formal Hardware Verification Methods: A Survey. Formal Methods in System Designs, 1:151-238, 1992.

17. Joyce, J.J.: Multi-level verification of microprocessor-based systems. $\mathrm{PhD}$ thesis, University of Cambridge, Computer Laboratory, May 1990.

18. Kumar, R., Schneider, K., Kropf, T.: Structuring and automating hardware proofs in a higher-order theorem-proving environment. Formal Methods in System Design, 2(2):165-223, 1993.

19. Langevin, M., Cerny, E.: Verification of processor-like circuits. In P. Prinetto and P. Camurati, ed., Proc. of Work. on Correct Hardware Design Methodologies, North-Holland, 1991.

20. Langevin, M., Cerny, E.: Comparing generic state machines. In K. Larsen and A. Skou, ed., Proc. of Work, on Computer-Aided Verification, Springer-Verlag, 1991.

21. Langevin, M., Cerny, E.: An extended OBDD representation for extended FSMs. In Proc. of EDAC-ETC-EUROASIC, 1994.

22. Loewenstein, P.N., Dill, D.L.: Verification of a multiprocessor cache protocol using simulation relations and higher-order logic. Formal Methods in System Design, 1:355-383, 1992.

23. Pierre, L.: The formal proof of sequential circuits described in CASCADE using the Boyer-Moore theorem prover. In L. Claesen, ed., Proc. of Int. Work. on Applied Formal Methods for Correct VLSI Design, North-Holland, 1989.

24. Staunstrup, J., Garland, S.J., Guttag, J.V.: Mechanized verification of circuit descriptions using the Larch Prover. In V. Stavridou, et al, ed., Proc. of Int. Conf. on Theorem Provers in Circuit Design: Theory, Practice and Experience, North-Holland, pp. 277-299, 1992.

25. Touati, H.J., Savoj, H., Lin, B., Brayton, R.K., Sangiovanni-Vincentelli, A.: Implicit State Enumeration of Finite State Machines Using BDDs. In Proc. of ICCAD, pp. 130-133, 1990.

26. Wolper, P.: Expressing interesting properties of programs in propositional temporal logic. In Proc. of the Symp. on Principles of Programming Languages, 1986.

27. Zhou, Z., Song, X., Corella, F., Cerny, E., Langevin, M.: Partitioning transition relation automatically and efficiently. In Proc. of Great Lakes Symp. on VLSI, 1995.

28. Zhou, Z., Song, X., Corella, F., Cerny, E., Langevin, M., Description and verification of RTL designs using multiway decision graphs. In Proc. of CHDL, 1995. 